Symposium : Hematology/Oncology-Part II Indian J Pediatr 1998; 65 : 805-814

\title{
Cell Cycle Control and Cancer
}

\author{
Hans Peter Wagner \\ University of Berm, Bern, Switzerland
}

\begin{abstract}
This review consists of two parts. In the first part normal mechanisms regulating the progression of cells through the cell cycle are briefly reviewed. Besides mitogenic stimulation, cyclin kinase inhibition, the G1 restriction point and the prb pathway, accuracy of DNA replication and DNA repair, the G2 to $M$ transition, apoptosis and the p 53 pathway, proteolytic, in particular ubiquitin dependent mechanisms involved in the initiation of DNA synthesis in the separation of sister chromatids and in the telophase to GO/G1 transition, are discussed. In the second part oncogene and tumor suppressor gene products are briefly characterized. Aberrations of cell cycle control mechanisms associated with cancer are grouped as follows : deregulation of protooncogenes by translocations juxtaposing protooncogenes to immunoglobulin - or $T$ cell receptor genes; translocations producing chimeric proteins unique to cancer cells; inversions and amplifications resulting in over expression of regulator genes; and deletions and mutations of tumor suppressor genes. It is emphasized that cancer is the result of a multistep process and that uncontrolled cell production and other alterations are, as a rule, late phenomena. (Indian J Pediatr 1998; 65 : 805-814)
\end{abstract}

Key words : Cell cycle; Regulator proteins; Checkpoints; Cancer; Chromosomal aberrations.

Cancer research has contributed significantly to a better understanding of basic biological phenomena such as cell division, cell differentiation and apoptosis. By studying pathologic alterations important informations on the structure, function and regulation of normal cells have been obtained. In this review the focus will be on cell cycle control mechanisms and on control deficits associated with cancer.

\section{Normal Cell Cycle Control Mechanisms}

Cell replication is a complex, well controlled process ${ }^{1,2}$. In each phase of the cell cycle, cells that are not prepared to enter the next phase are arrested at checkpoints, synonymous to blocks controlled by sur-

Reprint requests : H.P. Wagner, M.D., Professor of Pediatrics, Schneiderstrasse 45, 3084 Wabern, Switzerland veillance mechanisms ${ }^{2,3,4}$. In this way highfidelity replication of the approximately 3 billion base pairs during the $S$ phase is achieved and precise segregation of the newly formed chromosomes is guaranteed.

\section{Mitogenic stimulation}

The initiation of replication depends on extracellular signals. Receptors at the cell surface bind ligands and activate intracellular signal transmission pathways to the nucleus. Other ligands may block receptors and preclude signal transmission. The nucleus of a cell has to receive a series of signals within a certain time, before replication is initiated. Under sustained mitogenic stimulation a cell prepares itself for replication ${ }^{5,6}$. No or insufficient preparation occurs, if the cell is dif- 
ferentiated or if there is a lack of nutrients. Cells completely unprepared to divide but able to replicate are called GO cells; cells partially prepared are in early G1.

Early G1 is characterized by the fact that extracellular mitogenic stimuli can turn on, probably by regulating the activity of nuclear transcription factors such as c-fos, cjun, c-myc and others, the synthesis of cyclin dependent kinases (cdks). These kinases coordinate the transition of cells through the 4 phases of the cell cycle G1, S, G2 and $M^{24}$. In order to be active the cdks have to complex with cyclins and the holoenzyme has to be activated by the cdk activating kinase $C A K$, a multi-subunit enzyme consisting of cyclin $\mathrm{H}$ and cdk7 (= MO 15) ${ }^{78}$. CAK plays an important role in the regulation of transcription. In mammalian cells CAK is part of TFIIH, a basal transcription factor containing helicase, ATPase and nucleotide-excision repair activities. TFIIH is able to phosphorylate the carboxy-terminal domain (CTD) of RNA polymerase $\mathrm{II}^{9,10}$ Mitogens can only stimulate the synthesis of D-type cyclins. All other cyclins are synthesized at specific points during the cell cycle independent of extracellular stimuli.

\section{Cyclin kinase inhibitors}

The formation of active cyclin D - cdks can be blocked in many ways. Cdk inhibitors of the $\mathrm{p} 21^{\mathrm{WAF} 1 / \mathrm{CIP1}} \mathrm{p} 27^{\mathrm{KIP} 1}$ or $\mathrm{p} 57^{\mathrm{KIP} 2}$ or of the $\mathrm{p} 16^{\mathrm{INKAa}}, \mathrm{p} 15^{\mathrm{INK} 4 b}, \mathrm{p} 18^{\mathrm{INK} 4 \mathrm{c}}$ or $\mathrm{p} 19^{\mathrm{INK} 4 \mathrm{~d}}$ type bind to the cdks and block either the holoenzyme formation with cyclin or the phosphorylation at threonin 172 , by CAK? INK4 inhibitors of cdk4 antagonize only cyclin D - p21 ${ }^{\mathrm{CIP} 1}, \mathrm{p} 27^{\mathrm{KIP} 1}$ and $\mathrm{p} 57^{\mathrm{KIP} 2}$ antagonize cyclin D-and cyclin E- or A-dependent kinases ${ }^{11}$. The $\mathrm{p} 21^{\mathrm{CP} 1}$ is of particular interest since it binds also to the prolif- erating cell nuclear antigen (PCNA) which is a subunit of the DNA polymerase delta enzyme complex ${ }^{12}$

\section{G1 restriction point}

Active cyclin D-cdks phosphorylate the retinoblastoma tumor suppressor protein $\mathrm{prb}^{13}$. In the unphosphorylated state, the prb and the prb-related proteins p107 and p130 bind to transcription factors such as E2F and abl and arrest cells in G1. After phosphorylation these transcription factors are released and activate genes, the products of which are required for the transition from $G 1$ to $S$, such as dihydrofolate reductase, DNA polymerase alpha, thymidine kinase and others ${ }^{14}$. With the phosphorylation of the prb the restrictionor-checkpoint in G1 is passed and from there on, cell replication proceeds without additional mitogenic stimulation.

Both extra-and intracellular stimulatory and inhibitory factors are therefore integrated before the cell replication machinery is irrevocably initiated. Once it is started, the cell either divides or dies.

\section{Initiation of DNA synthesis}

Towards the end of G1 the pre-replication complex (pre-RC) is assembled at DNA replicating origins ${ }^{2,15}$. This complex represents a DNA replication licensing system assuring that each DNA sequence is replicated only one time before chromosome segregation $^{16}$. The pre-RC is composed of the origin recognition complex (ORC), an unstable protein called pcdc6, and the minichromosome maintenance proteins (mcms)

After phosphorylation of prb, ESF-DP heterodimers trigger the expression of cyc- 
lin $E$ and probably of cyclin A. The activation of cyclin E-and cyclin A-cdk2 initiates DNA synthesis and inhibits any de novo formation of pre- $\mathrm{RC}^{17}$. Another cyclin-cdk independent kinase, cdc7p-dbf4p (DDK) also interacts with components of the pre$\mathrm{RC}^{15}$.

After initiation of DNA synthesis, cyclin $E$ is inactivated by ubiquitin-dependent proteolysis (see below), and the DP components of heterodimeric ESFs are phophorylated by cyclin A-cdk2, precluding DNA binding of E2Fs". Cyclin A synthesis proceeds throughout $S$ and reaches peak values in G2. In early S. cyclin A is associated with cdk2, later with cdc2.

\section{Accuracy of DNA replication and DNA re- pair}

The accuracy of DNA replication is checked by proofreading functions coupled to the DNA polymerization activities. TFIH (see above) plays an important role ${ }^{18}$.

The gene ATM (= ataxia telangiectasia muted gene) appears to be involved in sensing DNA damage induced by irradiation and other agents. It activates the TP53 gene on $17 \mathrm{p} 13^{19.21} \cdot \mathrm{p} 53$ acts as a transcription factor ${ }^{22}$ p53 is phoshorylated specifically by cyclin A - cdk2 and cyclin B-cdc2, but not by cyclin D1-cdk4 or cyclin E-cdk2. Phosphorylation dramatically stimulates the binding of p53 to its targets in the p21 ${ }^{\text {WAF1/CIP1 }}$ and GADD45 genes ${ }^{10}$. p53 induces a block at the G1-S checkpoint and slows the progression of cells in S and G2 ${ }^{11}$. p53 also promotes apoptosis ${ }^{23,24}$.

Incomplete replication of telomeres is counteracted by telomerase, an enzyme that may be lost during differentiation. Telomeres, GC-rich sequences at the ends of each chromosome, are not completely copied during DNA synthesis, because DNA polymerases require an RNA primer to start and because they proceed only in the $5^{\prime}$ to $3^{\prime}$ direction ${ }^{25}$. Without telomerase, the potential of a cell to divide is limited (cell aging) ${ }^{26,22}$.

\section{G2 to $M$ transition}

The transition from $G 2$ to $M$ is also orchestrated by cyclin-dependent kinase activities, mainly cyclin B-cdc2. Cyclin B is first synthesized in late $S$ and peaks in late G2 or early $\mathbf{M}^{11}$. Cyclin B-cdc2 is activated by phosphorylation at threonine 161 . Many other factors, e.g. tyrosine kinases and phosphatases, control cyclin B-cdc2 activity. During mitosis active cyclin B-cdc2 phosphorylates various substrates, e.g. Btype nuclear envelope lamins, at mitosisspecific sites in the $\mathrm{N}$-terminal domain. Phosphorylation of these sites induces lamina depolymerization and nuclear membrane dissolution ${ }^{28}$. Cyclin B-cdc2 is also involved in the repression of RNA polymerase III $^{10}$

\section{Ubiquitination, sister chromatid separa- tion and telophase to G0/G1 transition}

Mitotic cyclins control the condensation of chromosomes, the spindle formation and the alignment and attachment of all pairs of sister chromatids to the mitotic spindle. For the latter process surveillance mechanisms exist detecting sister kinetochores which have not been properly aligned, ${ }^{47}$. These mechanisms block the activation of the anaphase-promoting complex (APC) or cytosome, a large multisubunit complex essential for chromosome splitting and destruction of M-phase cyclins ${ }^{2,17}$. 
APC and other proteolytic cell cycle enzymes target substrates for $26 \mathrm{~S}$ proteasome degradation by ubiquitination, i.e. assembling an ubiquitin chain on the substrate. Proteolytic degradation of the PDS1 and CUT2 proteins is essential for sister chromatid separation, and ubiquitinisation of M-phase cyclins for deblocking both the telophase to G0/G1 transition and the pre$\mathrm{RC}$ assembly ${ }^{17}$.

\section{Cell cycle exits}

Cells exit from the cell cycle either by differentiation or by necrotic or apoptotic cell death. Differentiation is probably restricted to cells in G0/early G1; death can occur in any phase of the cell cycle. Apoptosis is an energy-dependent programmed cell death in response to certain stimuli, associated with characteristic morphologic and biochemical features. Apoptosis can be induced by irradiation, chemotherapy, viral infections, cytotoxic lymphocyte killing, and growth factor or hormone withdraw$\mathrm{al}^{29-32}$. Some genes active in the cell cycle appear to influence apoptosis ${ }^{29-32}$ e.g. cmyc, p53 and prb24. Apoptosis is another mechanism regulating cell production ${ }^{33}$.

\section{Aberrations of Cell Cycle Control Mecha- nisms Associated with Cancer}

In solid tumors very complex chromosomal anomalies are found, even if primary tumours are examined : hyperdiploidy to near tetraploidy, structural abnormalities concerning the centromeric regions, amplifications, loss of chromosomes, deletions and mutations. Mutations in genes controlling checkpoints can relieve arrest signals and result in continued, uncontrolled progression through the cell cycle.
A dysregulation of cell cycle control is virtually pathognomic of all cancer cells.

\section{Oncogenes and Tumor Suppressor Genes}

Oncogenes and tumor suppressor genes have been identified by studying cancercausing viruses, by assessing cancer genes in tissue cultures, by analyzing genes at sites of chromosomal aberration in neoplastic cells and by isolating genes for cancer-predisposing familial syndromes.

Oncogene products or products of inappropriate or mutated protooncogene expression increase cell production. They function as growth factors [e.g. as plateletderived growth factor (PDGF)], growth factor receptors [e.g. for epidermal growth factor (EGFR)], proteins involved in signal transduction (e.g. upregulating RAS-related signaling pathways), transcription factors (e.g. c-myc), or antiapoptosis proteins (e.g. BCL-2). Overexpression of oncogenes can be the result of gene amplification, translocations or inversions. If germ line cells are affected, inheritance is dominant ${ }^{34}$.

Tumor suppressor gene products, e.g. prb, p53, p16 and apoptosis promoting proteins such as bax, reduce or block cell production. Tumor suppressor genes can be lost or mutated or their products can be inactivated, e.g. by viral oncoproteins of adenovirus (E1A), SV40 (large T antigen) and human papilloma-viruses (E6 and E7). If germ line cells are affected, inheritance is recessive (although some mutants may be dominant $)^{35}$.

\section{Chromosomal Aberrations in Cancer and Cell Cycle Control}

How frequently structural rearrangements occur under normal circumstances is 
unknown. Inherent genetic instability or fragile sites may mediate rearrangements. Chromosomal rearrangements are present in virtually all malignant cells. A number of specific cytogenetic abnormalities have been recognized that are closely or even uniquely associated with clinically distinct subsets of leukemias or solid tumors.

\section{Translocations Causing Deregulation of Gene Expression}

Chromosomal translocations can lead to a deregulation of gene expression, either an aberrant expression in a tissue that does not normally express the gene, or an overexpression. Examples are the translocations involving immunglobulin (Ig) or T-cell receptor (TCR) genes ${ }^{36}$.

In Burkitt's lymphoma and B-cell ALL the MYC gene at 8q24 is juxtaposed to an Ig gene, either the heavy chain gene at $14 \mathrm{q} 32$, the $\mathrm{k}$ light chain gene at $2 \mathrm{p} 12$, or the $\lambda$ light chain gene at $22 q 11$, by an $8 ; 14$, an $2 ; 8$ or an $8 ; 22$ translocation, respectively. This juxtaposition results in an abnormal regulation of MYC expression. The c-myc protein localizes to the nucleus and interacts, by heterodimerization, with MAX and other regulators of the myc translational system. Transcription from c-myc target genes is activated by MYC - MAX and is repressed by MAD-MAX or MXI-MAX heterodimers ${ }^{37+40}$. A c-myc activation stimulates cell proliferation in the absence of growth factors. In cells under nutrient deprivation, c-myc induces apoptosis. In quiescent cells c-myc expression results in activation of cyclin $E$ and $A$, while cyclin $D$ may be repressed. In signaling pathways cmyc is an esser.ial component, mediating transformation by oncoproteins such as bcr-abl, CSF-1 receptor tyrosine kinase and
RAS.

In follicular lymphoma the BCL2 gene on 18q21 is juxtaposed by translocation to the $\lg$ heavy chain gene at $14 \mathrm{q} 32$. The resulting chimeric protein blocks apoptosis ${ }^{36}$.

In B lineage mantle cell lymphomas $t(11 ; 14)(q 13 ; q 32)$ puts the $\lg$ heavy chain inducer on $14 q 32$ into the cyclin D1 locus leaving the $\mathrm{B} 1$ coding sequences uninterrupted ${ }^{11}$. While normal B lymphocytes express only cyclins D2 and D3, all lymphoma cells with a $t(11 ; 14)$ ectopically synthesize cyclin D1 (cyclin D1 overexpression in other solid tumors : see below).

In T-cell disorders, TCR $\beta$-and $\delta$-chain; $\beta$; cr $\gamma$-chain genes at $14 q 11 ; 7 q 34-35$ and $7 \mathrm{p} 15$, respectively, are juxtaposed by translocations to genes encoding transcription factors, such as TAL1, LYL1 and TAL2 ${ }^{36,41}$.

\section{Translocations Producing New Fusion (chimeric) Proteins}

Translocations can produce novel fusion proteins resulting from the juxtaposition of coding sequences normally located on different chromosomes. Fusion proteins are only observed in the cancer cells and may therefore be important in diagnosis.

\section{Leukemias}

Examples for fusion proteins are the bcr/abl protein [CML, $t(9 ; 22)$ ], the AML 1/ETO protein [AML type M2 (M 4), $t(9 ; 21)$ ], the PML/RARA protein [AML type $M 3, t(15,17)]$, the $E 2 A / P B X 1$ protein [pre-B ALL, $t(1 ; 19)$ ] and a number of fusion proteins resulting from translocations involving the MLL gene at $11 \mathrm{q} 23$ [e.g. AML type M 5, $t(9 ; 11)$ ] infant ALL, $t$ 
$(4 ; 11)]^{36}$. The $p 210^{\text {bcr-sbl }}$ is located on the cytoplasmic surface of the cell membrane and transmits growth-regulatory signals from the cell surface receptors via the ras signal transduction pathway to the nucleus ${ }^{42}$. Tyrosine kinase inhibitors inhibiting the growth of cells expressing bcr-abl or related fusion proteins have been described ${ }^{43}$. The core binding factor $\alpha(C B F \alpha)$, the normal product of AML1, complexes with the core binding factor $\beta$ (CBF $\beta$ ) to form a transcription factor regulating the expression of genes critical to myeloid cell growth, differentiation and function such as IL3, GMCSF, MPO and CSF 1 receptor. The AML1/ ETO protein binds to the regulatory regions of these genes but cannot activate their expression 4,15 .

The PML/RARA protein is released on exposure to all-trans-retinoic acid and activates retinoic acid responsive gene transcription inducing differentiation of APL cells $^{35,4,46}$.

E2A, the product of a gene on $19 \mathrm{q} \mathrm{13}$, functions as a transcription factor. The gene on 1q21 is a homeobox gene. In the fusion protein the DNA-binding protein of E 2 A is replaced by that of the PBX 1 gene which may alter the target genes of the chimeric transcription factor"

The MLL gene encodes a transcription factor with a zinc finger domain, a DNA binding AT-hook, a DNA methyltransferase domain and transcription activation and repression domains. In all fusion proteins with the MLL protein, the latter has lost the activation domain but retains the DNA binding and repression domains ${ }^{36,4,10,49}$,

\section{Solid tumors}

In a large proportion of rhabdomyosar- comas at $(2 ; 13)(q 35 ; q 14)$ creating a fusion transcription factor involving the PAX 3 homeobox gene on $2 q 35$ and the FKHR (forkhead domain) on $13 \mathrm{q} \mathrm{14}$, was observed. A variant, $t(1 ; 13)$ ( $p 36 ; q 14)$, was also described ${ }^{3650}$.

In Ewing's sarcoma, peripheral neuroepithelioma, Askin's tumor, esthesioneuroblastoma and a few medulloblastomas a $t(11 ; 22)(q 24 ; q 12)$ is observed. The translocation results in a chimeric transcription factor containing the transactivation domain of the EWS gene (22q12) and the DNA binding of the EL11 gene $(11 \mathrm{q} 24)^{35,51,52}$.

Inversions and Amplifications Leading to Overexpression of Cell Cycle Regulators or Protooncogenes

In parathyroid adenomas an i(11)(p15;q13) links cyclin D1 to parathyroid hormone ${ }^{33}$. Cyclin D1 (formerly PRAD1) encoded on 11q13 (formerly CCND1) and CDK4 encoded on 12q13 (together with the p53 antagonist MDM2) are overexpressed in sarcomas and gliomas ${ }^{54,55}$. Amplification of the D1 locus was observed in almost half of head and neck squamous cell carcinomas, in about a third of esophageal carcinomas and in bladder cancers, primary breast cancers, small-cell lung cancers and hepatocellular carcinomas ${ }^{56}$. Aberrant overexpression of cyclin D1 was also seen in sarcomas, colorectal tumors and melanomas even though $\mathrm{D} 1$ gene amplification frequencies were $l^{56} w^{56}$. In mice overexpression of D1 in mammary epithelial cells leads to hyperproliferation and tumor formation, while animals nullizygous for D1 show severe defects in mammary lobuloalveolar development during pregnancy ${ }^{57,58}$. 
In neuroblastomas predominantly deletions or rearrangements of the terminal portion of $1 \mathrm{p}$ are found. Loss of heterozygosity for 1p36.2-3. was preseent in $26 \%$ of primary neuroblastomas ${ }^{59}$. In up to a third of the cases amplification of the N-MYC oncogene is observed ${ }^{60}$.

\section{Deletions and Mutations of Tumor Sup- pressor Genes}

Retinoblastoma is the hallmark of a tumor due to the loss of a tumor suppressor gene, the RB1 gene on 13q14. Both alleles have to be deleted, before retina cells become neoplastic. Inactivation of the RB gene is also seen in osteosarcoma, carcinoid tumors, small-cell lung cancers and invasive bladder cancers ${ }^{61}$. As indicated previously, the RB product, prb, can also be inactivated by viral oncoproteins, e.g. human papillomavirus protein $E 7^{61,62}$.

Inactivation of the INK4a locus (on 9p21) by homozygous deletion, methylation of the promotor or point mutation occurs in approximately $50-60 \%$ of gliomas, head and neck carcinomas, mesotheliomas and T-All, in about $25-50 \%$ of bladder and pancreas cancer and melanoma as well as in oesophagus, gastric, non-small-cell lung, renal and prostate cancers ${ }^{56,63}$. Mutations of the INK4a (=CDKN2 or MTS1) gene on 9 p21 resulting in production of inactive p16 $6^{\text {INKan }}$, are associated with familial melanoma and are seen in a high proportion of biliary tract and esophageal carcino$\mathrm{mas}^{64,65} \cdot \mathrm{p} 16$ is frequently detected in premalignant lesions. It was suggested, therefore, that p16 inactivation represented an early event in tumor development ${ }^{66} . \mathrm{p} 16$ is also inactivated by human papillomavirus protein E6 $6^{62}$. Loss of p16 ${ }^{\text {INK4a }}$ might mimic cyclin D1 or CDK4 overexpression, both leading to hyperphosphorylation and inactivation of prb.

The TP53 gene on $17 \mathrm{p} 13$ is the most frequently mutated gene in cancer ${ }^{67}$. Loss of TP53 gene alleles, or mutations associated with nuclear accumulation of missense p53, are characteristically seen in more advanced cancers. In patients with colorectal cancer, up to $80 \%$ were found to have deletions of $17 \mathrm{p} 13$ and the rest had mutations. In breast cancer, up to $50 \%$ had losses or mutations of the p17p13 gene and in many other cancers such as lung and liver, head and neck, pancreas, oesophageal, ovarian, endometrial and cervical cancers as well as lymphomas, sarcomas and brain tumors nuclear accumulation of missense p53 was found by immunostaining. By examining the spectrum of $\mathrm{p} 53$ mutations in a series of tumors, environmental or endogenous factors involved in tumorigenesis might be recognized ${ }^{68}$.

Cancer is the result of a multistep process. If, e.g., chromosomal translocations result in a loss of checkpoint control and uncontrolled proliferation, the increased cell production can still be compensated by increased apoptotic death. Only if a second mutation blocks apoptosis, decompensation and accumulation of malignant cells occurs. As genetic instability increases due to diminished cell cycle control, the malignant cells may acquire additional characteristics such as invasiveness and decreased drug sensitivity.

\section{Conclusions}

This review demonstrates that human cancers are often associated with inherited or acquired chromosomal aberrations leading to overexpression or repression of genes that play an important role in cell cy- 
cle replication, differentiation and/or apoptosis. It is also evident, that important decisions, e.g. the triggering of cell division or of apoptosis, or the induction of cell differentiation, are subject to very complex steering mechanisms, that take into account a multitude of extrinsic and intrinsic factors. It is not astonishing, therefore, that cancer does not result from one, but from at least two or more genetic defects or events. There is good evidence to suggest that cancer develops stepwise, and that uncontrolled cell proliferation as well as invasiveness and metastatic spreading or the development of drug resistance, are, as a rule, late phenomena of a long lasting process. It is therefore not surprising, that older individuals are more often affected than younger ones. By extending our understanding of basic biological phenomena, molecular biology and genetics are poviding bases for more rational diagnostic and therapeutic approaches, by offering alternatives to actual strategies and by defining promising targets for new therapeutic efforts.

\section{REFERENCES}

1. Murray AW, Kirschner MW. Dominoes and clocks : the union of two views of the cell cycle. Science $1989 ; 247: 614-621$.

2. Nasmyth K. Viewpoint. putting the cell cycle in order. Science 1996; 274 : 16431645.

3. Hartwell LH, Weinert TA. Checkpoints : Controls that ensure the order of cell cycle events. Science 1989; $246: 629-634$.

4. Elledge SJ. Cell cycle checkpoints : preventing an identity crisis. Science 1996; 274: 1664-1672.

5. Pardee AB. G1 events and regulation of cell proliferation. Science 1989; $246: 603$ 608.

6. Aronson S. Growth factors and cancer.
Science 1991; 254 : 1146-1153.

7. Fisher RP, Morgan DO. A novel cyclin associates with MO15/cdk7 to form a cdkactivating kinase. Cell 1994; 78 : 713-724.

8. Morgan DO. Principles of cdk regulation. Nature 1995; 374 : 374 : 131-134.

9. Nigg EA. Cyclin dependent kinase 7 : at the crossroads of transcription, DNA repair and cell cycle control? Curr Opin Cell Biol 1996; 8 : 312-317.

10. Dynlacht BD. Regulation of transcription by proteins that control the cell cycle. $\mathrm{Na}$ ture 1997; 389 : 149-152.

11. Sherr CJ. Cancer cell cycle. Science 1996; 274 : 1672-1677.

12. Jacks $T$, Weinberg RA. The expanding role of cell cycle regulators. Science 1998; $280: 1035-10$

13. Weinberg RA. The retinoblastoma protein and cell cycle control. Cell 1995; $81: 323$ 330.

14. Dyson N. pRB, p107. and the regulation of the E2F transcription factor. / Cell Sci (suppl) 1994; 18 : 81-87.

15. Stillmann B. Cell cycle control of DNA replication. Science 1996; 274 : 1659-1664.

16. Chong JPJ, Mahbubani HM, Khoo C-Y, Blow JJ. Purification of an MCM-containing complex as a component of the DNA replication licensing system. Nature 1995; 375 : 418-421.

17. King RW, Deshaies RJ, Peters J-M, Kirschner MW. How proteolysis drives the cell cycle. Science 1996; 274 : 1652-1659.

18. Sancar A. Mechanisms of DNA excision repair. Science 1994; 266 : 1954-1956.

19. Brown L, McCarthy N. A sense-abl response? Nature 1997; $387: 450-451$.

20. Baskaran R, Wood LD, Whitaker LL et al. Ataxia telangiectasia mutant protein activates c-able tyrosine kinase in response to ionizing radiation. Nature 1997; 387 : 516519.

21. Vogelstein B, Kinzler KW. 553 function and dysfunction. Cell 1992; $70: 523-526$.

22. Prives C, Manfredi JJ. The $p 53$ tumor suppressor protein. Genes Dev 1993; $7: 529$. 534. 
23. Wyllie A. Apoptosis. Clues in the p53 murder mystery. Nature 1997; 389 : 237 238.

24. Polyak K, Xia Y, Zweier JL, Kinzler KW, Vogelstein B. A model for p53-induced apoptosis. Nature 1997; 389 : H 300-305.

25. Greider CW, Blackburn EH. Telomeres, telomerase and cancer. Sci Amer 1996; 274 (2) : 80-85.

26. De Lange T. Telomeres and senescence : ending the debate. Science 1998; 279 : 334335.

27. Bodnar AG, Quelette M, Frolkis $M$ et al. Extension of life-span by introduction of telomerase into normal human cells. Science 1998; 279 : 349-352.

28. Peter M, Nakagawa J, Doree M, Labbé JC, Nigg EA. In vitro disassembly of the nuclear lamina and $M$ phase-specific phosphorylation of lamins by cdc2 kinase. Cell 1990; 61 : 591-602.

29. Duke RC, Ojcius DM, Young JD-E. Cell suicide in health and disease. Sci Amer 1996; 274 (6) : 48-55.

30. Nagata $S$, Goldstein $P$. The fas death factor. Science 1995; 267 : 1449-1456.

31. Chinnaiyan AM, Chaudhary D, O'Rourke K, Koonin EV, Dixit VM. Role of CED-4 in the activation of CED-3. Nature 1997; 388 : 728-729.

32. Irmler $M$, Thome $M$, Hahne $M$ et al. Inhibition of death receptor signals by cellular FLIP. Nature 1997; 388 : 190-195.

33. Reed JC. Double identity for proteins of the Bcl-2 family. Nature 1997; 387 : 773776.

34. Krontiris TG. Oncogenes. $N$ Engl J Med 1995; 333 : 303-306.

35. Weinberg RA. Tumor suppressor genes. Science 1991; 254 : 1138-1146.

36. Rabbits TH. Chromosomal translocations in humna cancer. Nature 1994; $372: 143$ 149.

37. Gu W, Cechova K, Tassi V, Della-Favera R. Opposite regulation of gene transcription and cell proliferation by c-myc and Max. Proc Natl Acad Sci 1993; 90 : 29352939.
38. Ayer D, Kretzner L, Eisenmann R. Mad, a heterodimeric partner for Max that antagonizes Myc transcriptional activity. Cell 1993; $72:$ 211-222.

39. Zervos A, Gyuris J, Brent R. Mxi, a protein that specifically interacts with Max to bind Myc-Max recognition sites. Cell 1993; 72 : 223-232.

40. Amati B, Brooks $\mathrm{M}$, Levy $\mathrm{N}$ et al. Oncogenic activity of the c-myc protein requires dimerization with Max. Cell 1993; 72 : 233-245

41. Uckun FM, Sensel MG, Sun L et al. Biology and treatment of childhood T-lineage acute lymphoblastic leukemia. Blood 1998. 91 : 735-746.

42. Lugo TG, Pendergast AM, Muller AJ, Witte ON. Tyrosine kinase activity and transformation potency of bcr-able oncogene products. Science 1990; 247 : 1079-1082.

43. Carroll M, Ohno-Jones S, Tamura $S$ et al. CGP 57148, a tyrosine kinase inhibitor inhibits the growth of cells expressing BCRABL, TEL-ABL, and TEL-PDGFR fusion proteins. Blood 1997; 90 : 4947-4952.

44. Tenen DG, Hromas R, Licht JD, Zhang DE Transcription factors, normal myeloid development and leukemia. Blood 1997; 90 : 489-519.

45. Okuda T, Cai Z, Yang S et al. Expression of a knocked-in AML1-ETO leukemia gene inhibits the establishment of normal finitive hematopoiesis and directly generates dysplastic hematopoietic progenitors. Blood 1998; 91 : 3134-3143.

46. Benedetti L, Levin AA, Scicchitano BM et al. Characterization of the retinoid binding properties of the major fusion products present in acute promyelocytic leukemia cells. Blood 1997; 90 : 1175-1185.

47. Hunger SP. Chromosomal translocations involving the E2A gene in acute lymphoblastic leukemia : clinical features and molecular pathogenesis. Blood 1996; 87 : 1211-1224.

48. Poirel H, Rack K, Delabesse E et al. Incidence and characterization of MLL gene 
(11q23) rearrangements in acute myeloid leukemia M1 and M5. Blood 1996; 87 : 2496-2505.

49. Behm FG, Raimondi SC, Frestedt IL et al. Rearrangement of the MLL gene confers a poor prognosis in childhood acute lymphoblastic leukemia, regardless of presenting age. Blood 1996; 87 : 2870-2877.

50. Kelly KM, Wamer RB, Sorensen PHB, Xiong QB, Barr FG. Common and variant gene fusions predict distinct clinical phenotypes in rhabdomyosarcoma. I Clin Oncol 1997; 15 : 1831-1836.

51. Delattre O, Zucman J, Melot $T$ et al The Ewing family of tumors - a subgroup of small-round-cell tumors defined by specific chimeric transcripts. $N$ Engl J Med 1994; 331 : 294-299.

52. Zoubek A, Dockhorn-Dwornizak B, Delattre $\mathrm{O}$ et al. Does expression of different EWS chimeric transcripts define clinically distinct risk gorups of Ewing tumor patients? J Clin Oncol 1996; 14 : 1245-1251.

53. Motokura T, Bloom T, Kim HG et al. A novel cyclin encoded by a bcl1-linked candidate oncogene. Nature 1991; 350 : 512-515.

54. Khatib ZA, Matsushime $H$, Valentine $M$, et al. Coamplification of the CDK4 gene with MDM2 and GL1 in human sarcomas. Cancer Res 1993; 53 : 5535-5541.

55. He J, Allen JR, Collins VP et al. CDK4 amplification is an alternative mechanism to p16 gene homozygous deletion in glioma cell lines. Cancer Res 1994; 54 : 5804-5807.

56. Hall M, Peters G. Genetic alterations of cyclins, cyclin-dependent kinases and cdk inhibitors in human cancer. Adv Cancer Res 1996; 68 : 67-108.

57. Wang TC, Cardiff RD, Zuckerberg $L$ Lees E, Arnold A, Schmidt EV. Mammary hyperplasia and carcinoma in MMTYcyclin D1 transgenic mice. Nature 1994; $369: 669-671$.
58. Sicinski P, Donaker JL, Parker SB et al. Cyclin D1 provides a link between development and oncogenes in the retina and breast. Cell 1995; $82: 621-630$.

59. White PS, Maris JM, Beltinger C et al. A region of consistent deletion in neuroblastoma maps within 1p36.2-2.3 Proc Natl Acad Sci USA 1995; 92 : 5520-5524.

60. Tonini GP, Boni L Pession A. et al. MYCN oncogene amplification in neuroblastoma is associated with worse prognosis, except in stage 4s; the Italian experience with 275 children. J Clin Oncol 1997; 15 : 85-93.

61. Sellers WR, Kaelin Jr WG. Role of the retinoblastoma protein in the pathogenesis of human cancer. J Clin Oncol 1997; 15 : 3301-3312.

62. Alani RM, Munger K. Human papillomavirus and associated malignancies. J Clin Oncl 1998; $16:$ 330-337.

63. Liggett JR WH, Sidransky D. Role of the p16 tumor suppressor gene in cancer. $J$ Clin Oncol 1998; 16 : 1197-1206.

64. Hussussian CJ, Struewing JP, Goldstein AM et al. Germline p16 mutations in familial melanoma. Nature Genet 1994; 8 : 15-21.

65. Goldstein AM, Fraser MC, Struewing JP et al. Increased risk of pancreatic cancer in melanoma-prone kindreds with $16^{1 \mathrm{~N}}$ mutations. N Engl J Med 1994; 333 : 970974.

66. Reznikoff CA, Yeager TR, Belair CD et al. Elevated p16 at senescence and loss of p16 at immortalization in human papillomavirus $16 \mathrm{E6}$, but not E7, transformed human uroepithelial cells. Cancer Res 1996; 56 : 2886-2890.

67. Hollstein M, Sidransky D, Vogelstein B, Harris C. p53 mutations in human cancer. Science 1991; $253:$ 49-53.

68. Harris CC, Hollstein M. Clinical implications of the p53 tumor-suppressor gene. $N$ Engl J Med 1993; 1318-1327. 